\title{
EL PAPEL DE LA UNIVERSIDAD EN EL MARCO DE UNA SOCIEDAD EN EMERGENCIA
}

\section{THE ROLE OF THE UNIVERSITY IN THE FRAMEWORK OF A SOCIETY IN EMERGENCY}

\section{Claudia Esperanza Saavedra Bautista1}

\section{Claudia Figueroa2}

\section{Iván Darío Mejía Ortega3}

Colombia

\section{RESUMEN}

Este aporte teórico esboza una postura reflexiva en torno a los principales desafíos y provocaciones que enfrenta la universidad en la actualidad producto de las apuestas

1 Doctorando en ciencias de la educación, Docente de la Licenciatura en Tecnología, Facultad Seccional Duitama, Universidad Pedagógica y Tecnológica de Colombia, Duitama, Colombia, claudia.saavedra@uptc.edu.co, Orcid: https://orcid.org/0000-0002-7981-4378

2 Doctora en ciencias de la educación, Docente de la Licenciatura en Psicopedagogía, Universidad Pedagógica y Tecnológica de Colombia, Tunja, Colombia, claudia.figueroa01@uptc.edu.co, Orcid: https://orcid.org/0000-00034185-2923

3 Doctorando en ciencias de la educación, Docente de la Licenciatura en Informática, Universidad Pedagógica y Tecnológica de Colombia, Tunja, Colombia, ivan.mejia@ uptc.edu.co, Orcid: https://orcid.org/0000-0003-2767-2395 tecnológicas emergentes que transitan en la sociedad presente. El análisis de este trabajo se desarrolla siguiendo la metodología de teoría fundamentada donde a partir de un muestreo teórico se analizaron diferentes artículos científicos para intentar develar la crisis de la universidad desde diferentes vertientes en el marco de una sociedad en emergencia. Los resultados se consolidan a partir de los procesos de discusión y reflexión a los que llegan los investigadores y permiten concluir que es preocupante percibir cómo la crisis financiera ha afectado la autonomía de la universidad pública precarizando su misión formativa e impacto en la sociedad, por lo que se hace necesario pensar en una universidad distanciada de los rankings y del mercado universitario para asumir la academia con compromiso social. 
PALABRAS CLAVE: Crisis, Desafíos, Sociedad, Tecnología, Universidad

\section{ABSTRACT}

This theoretical contribution outlines a reflective stance on the main challenges and provocations that the university currently faces as a result of the emerging technological bets that transit in today's society. The analysis of this work is carried out following the grounded theory methodology where, from a theoretical sampling, different scientific articles were analyzed to try to unveil the crisis of the university from different aspects within the framework of a society in emergency. The results are consolidated from the discussion and reflection processes that researchers reach and allow us to conclude that it is worrisome to perceive how the financial crisis has affected the autonomy of the public university by precarizing its formative mission and impact on society, so that it is necessary to think of a university distanced from the rankings and the university market to assume the academy with social commitment..

KEY WORDS: Crisis, Challenges, Society, Technology, University

\section{INTRODUCCIÓN}

Los procesos de reflexión entre investigadores abren la posibilidad de discutir y analizar campos de estudio en torno a un interés común. Es así, que la escritura de este texto ha sido la oportunidad de reflexionar sobre los desafíos que afronta la universidad en tiempos emergentes. Este es un escrito que se desarrolla en el marco del desarrollo de una tesis doctoral y permite referir que la universidad como un escenario de construcción de conocimiento merece procesos de debate y reflexión que conduzcan a analizar los distintitos saberes que se reúnen para enfrentar la complejidad de la modernidad. En este marco, se instalan distintas culturas y lenguajes académicos que se constituyen como ejes primordiales para atender las problemáticas educativas actuales y adentrarse en discusiones que se encaminan a reconocer el papel de la universidad en el marco de los desafíos del siglo XXI. Sin duda, la universidad actualmente tiene una responsabilidad social que debe responder a los desafíos que la globalización señala, los cuales han ido apareciendo producto del apresurado avance tecnológico; por esta razón, se debe analizar los elementos que constituyen la formación de profesionales íntegros para identificar la coherencia con el contexto actual y las políticas educativas. Este análisis debe permitir revisar la labor que se realiza a nivel de la calidad y pertinencia del profesional que se está formando, como contribución con la sociedad. Tal como lo planteaba Dewey (1989), quién argumentaba que lo más difícil era lograr la relación entre escuela y sociedad.

En esta época el sistema educativo reclama soluciones novedosas que trascienden de la transmisión de conceptos a la promulgación de una didáctica innovadora, por esta razón la educación debe recobrar su sentido a través de la reconfiguración de la misma y con el objetivo de reconocer en la formación de profesionales una acción transformadora que impacte la sociedad de manera positiva para aportar a la reconstrucción del tejido social.

\section{PERSPECTIVA TEÓRICA}

La universidad desde sus inicios en el siglo XII se ha consolidado como un lugar para la promoción de conocimiento y la formación de profesionales que contribuyan en la solución de problemáticas sociales. "La globalización contra- hegemónica de la universidad como bien público, mantiene la idea de proyecto nacional, sólo que la concibe de un modo no nacionalista ni autárquico. En el siglo XXI sólo habrá naciones en la medida en que haya proyectos nacionales de cualificación de la inserción en la sociedad global" (De Souza, 2007, p. 16). A pesar que la universidad en sus inicios se 
fundamentó más en la promoción de títulos, que en la formación de profesionales con altas cualidades humanas; esta función se ha venido transformando al pasar de los años y ahora se presenta como un escenario con responsabilidad social, donde convergen intereses de docentes y estudiantes que se encaminan a atender problemáticas sociales; sin desconocer que en sus inicios predominaban intereses asociados con la teología, humanidades, leyes y ciencias sociales.

Según Bernheim, C. T. (2003) "La universidad latinoamericana ha crecido en el siglo XIX, como entidad ficticia, a expensas de las escuelas profesionalizantes, las cuales han producido diplomas incultos, pero técnicos, si es posible una técnica cabal sin el ineludible trasfondo de cultura general correspondiente". p.98. Por su parte, Brunner (1990), considera que la universidad está sufriendo grandes cambios como su masificación, donde miles de estudiantes de diversos contextos convergen en medio de un amplio y diverso abanico de carreras que ha llevado a que el acceso sea un proceso complejo que sin duda puede afectar los procesos educativos y administrativos que emana la universidad.

Como antecedente importante, vale la pena citar el30 de octubre de 1913cuando quedó constituida jurídicamente la Universidad Libre, como entidad que reemplazaría a la independiente, laica y civilista Universidad Republicana, por lo que la universidad Libre tuvo origen en la Universidad republicana. En la crisis nacional y en medio de las luchas partidistas, dos modelos de universidad se enfrentan en la culminación del siglo XIX. La universidad de la mentalidad tradicional fincada en la regeneración, educación ligada a la catolicidad y la universidad laica, originada en el planteamiento del liberalismo radical, que defiende la separación de la iglesia y el estado, la educación libre y laica. Las universidades han venido creciendo cada vez más y se han convertido en organismos con un tejido social más complejo y heterogéneo. Se ha propuesto que un nombre más apropiado para la universidad actual sería el de multiversidad" (Kerr, 1963, p. 19).

De Sousa (2007), por su parte, plantea qué hacer con la universidad del siglo XXI, mediada por la relación Sociedad - Estado en su capacidad de direccionamiento, la cual enmarca el sentido de lo público y los procesos de interacción cultural, intercultural, pluricultural; desde lo disciplinar e interdisciplinar, del trabajo del conocimiento universitario al pluriuniversitario, como soporte de una sociedad del conocimiento, de la ciencia, la tecnología y los procesos innovadores, principalmente, el trabajo en red que conlleva a propiciar cambios en la manera de orientar la educación superior, bases fundamentales y cambios contundentes "de hegemonía, de legitimidad, e institucional”.

Es claro que la historia de la universidad tiene avances y retrocesos, que se reflejan en desarrollos significativos en los procesos formativos en la profesionalización de un talento humano, en la creación y desarrollo de programas articulados al desarrollo tecnológico, científico, económico y social de cada nación. Así mismo, incursiona la mercantilización de la educación, la desfinanciación de la educación pública y otras problemáticas producto del neoliberalismo, que han conducido a fortalecer la crisis en la educación superior, la situación es crítica, ya que el sentido de lo público ha colapsado, principalmente, en países de tercer mundo o los que están en subdesarrollo y paupérrimos, lo cual está en desventaja con las universidades privadas. De hecho, el mismo sistema en su comprensión es complejo, difícil la situación de las mismas. De otra parte, De Sousa (2007) citado en Martínez et al. (2016), señala que "En estos países el sistema educativo en su conjunto- estuvo siempre ligado a la construcción del proyecto de nación, 
un proyecto nacional casi siempre elitista que la universidad debía formar...En el siglo XXI sólo habrá naciones en la medida en que existan proyectos nacionales de calificación de la inserción en la sociedad global. Para los países periféricos y semiperiféricos no hay calificación sin que la resistencia a la globalización neoliberal se traduzca en estrategias de globalización alternativa". (p.395).

Lo que la universidad hizo a partir de estas rupturas y cambios fue articular el conocimiento e ir mucho más allá de sus propias dinámicas, lograr reformarse a partir de una sociedad del conocimiento, permitiendo, como lo señala De Sousa (2007), comprometerse con cinco áreas de acción: acceso, extensión, investigación-acción, ecología de saberes, universidad y escuela pública. Las dos primeras son las más convencionales, pero deberán ser profundamente revisadas, la tercera ha sido practicada en algunas universidades latinoamericanas $\mathrm{y}$ africanas durante algunos períodos de mayor responsabilidad social por parte de la universidad, la cuarta constituye una decisiva innovación en la construcción de una universidad postcolonial, la quinta es un campo de acción que tuvo en el pasado una gran presencia pero que debe ser ahora reinventada.

Los resultados de esta crítica a la universidad apuntan a analizar algunos puntos claves, entre ellos: 1) ¿cómo se da el acceso a la educación en general y de qué manera incide en la apropiación de una educación pública y gratuita? 2) ¿cómo se está financiando la educación pública y bajo qué condiciones accede la población estudiantil? 3) ¿Cuál es el papel de los entes gubernamentales para favorecer a la clase pobre y media en los procesos de formación profesional y qué procesos de inclusión favorece? 4) ¿cuáles son las acciones de los Estados para elevar la calidad de vida de los universitarios y generar puestos de trabajo o formalizar los que existen? Realmente, las dimensiones de cobertura y acceso implican valorar el sentido para lo que fueron hechas las instituciones de educación superior y priorizar su desarrollo para garantizar una educación de calidad, equitativa, incluyente y formal. Ejemplos claros se tienen en Brasil, país que se sustenta en una educación pública e incluyente, tanto en sus procesos de profesionalización, como en los avances mismos de la universidad a nivel estructural, en el marco de una sociedad del conocimiento y unas expectativas de vida interesantes y pertinentes para los requerimientos de la sociedad del siglo XXI.

Para el caso colombiano, con la promulgación de la Ley 30 de 1992, se define como propósito central de la Educación Superior la formación integral, la calidad académica, la pertinencia social y académica. De igual manera, la ley 115 de 1994, en el artículo 112, define que las instituciones formadoras de educadores, corresponden a las universidades y a las demás instituciones de educación superior que posean una facultad de educación u otra unidad académica dedicada a la educación, la formación profesional, la de posgrado y la actualización de los educadores.

De la misma manera esta misma ley en su artículo 113 , señala que, con el fin de mantener un mejoramiento continuo de la calidad de los docentes, todo programa de formación de docentes debe estar acreditado en forma previa, de acuerdo con las disposiciones que fije el Consejo Nacional de Educación Superior, CESU, o el Ministerio de Educación Nacional, MEN, para el caso de las Normales Superiores.

De acuerdo a lo anterior, es significativo el aporte De Sousa (2007), cuando plantea que "EI principio que debe afirmarse debe ser el compromiso de la universidad con la escuela pública. A partir de ahí, se trata de establecer mecanismos institucionales de colaboración a través de los cuales se construya una integración 
efectiva entre la formación profesional y la práctica educativa". (p.73).

Realmente se trata de que las próximas reformas educativas a la educación superior según el mismo De Sousa, deben lograr una verdadera: 1) Valorización de la formación inicial y su articulación con los programas de formación continua. 2) Reestructuración de los cursos de licenciatura con el fin de asegurar la integración curricular entre la formación profesional y la formación académica; 3) Colaboración entre investigadores universitarios y profesores de instituciones educativas públicas en la producción y difusión del saber pedagógico, mediante el reconocimiento y el estímulo de la investigación-acción; 4) Creación de redes regionales y nacionales de universidades públicas para desarrollo de programas de formación continua en alianza con los sistemas de educación pública.

Es significativo el aporte de Ortiz (2017), cuando plantea en sus reflexiones y trabajos, principalmente en su obra "La Universidad emergente del siglo XXI", que esta avanza en el desarrollo de los procesos de enseñanza aprendizaje y los procesos curriculares. Así mismo, toca aspectos de las teorías del aprendizaje, los modelos pedagógicos y las tendencias pedagógicas en la universidad y sus propios desafíos del siglo $\mathrm{XXI}$, que se sitúan en apuestas tecnológicas que emergen en un escenario cambiante y diverso, donde confluyen jóvenes catalogados como generaciones emergentes, que llegan a implementar viejas estrategias en nuevos escenarios que se revisten de diversidad. Los jóvenes de ahora no solo buscan una profesión o una titulación, sino que reclaman propuestas novedosas que les permita entender su realidad e insertarse en una sociedad compleja. Según Pujadas (s.f), "La necesidad de competir y la exigencia de calidad en la enseñanza y la investigación requieren cada vez inversiones más costosas en laboratorios y en tecnología educativa”. (p.5).

De otra parte, surgen investigaciones que aportan al trabajo, y son aquellas que hacen parte de "las necesidades emergentes y responsabilidad social en la universidad", según De la Red Vega (2009), señala tres aspectos fundamentales que hay que tener en cuenta en la universidad: recursos económicos, recursos humanos y recursos sociales, como soporte de las necesidades. postula necesidades sociales emergentes, dando relevancia a la educación en: gestión, docencia, investigación y compromiso social, de la mano con la manera como se debe organizar la universidad en: desarrollo ciudadano, democrático y sostenible, inclusión social y la riqueza de las redes sociales.

Así mismo, Fernández (2014), presenta una investigación sobre los grandes retos que tiene la universidad a partir de la articulación entre "universidad, sociedad, conocimiento" sustentado en las demandas que trae la universidad del siglo XXI, pero también en la falta de políticas claras y coherentes desde la sociedad del conocimiento; llama la atención, cuatro aspectos fundamentales, que el autor resalta: la universidad que tenemos, la universidad y la sociedad: Su democratización, la universidad y el conocimiento: la innovación; vista desde la región en sus políticas públicas educativas desde las últimas décadas, su gobernabilidad y acciones desde cada gobierno de turno, caso específico de Argentina y su relación con América Latina.

De otra parte las apuestas que hace Corrales (2007) plantea que la universidad del siglo $\mathrm{XXI}$, tiene como misión, propender por una 1) generación de conocimiento útil para la vida individual y social tendiente a la elevación del nivel de conciencia propio de la sociedad creativa; 2) función inmunizadora del cuerpo social respecto de la información generativa errónea (Bohm,1988), y 3) investigación y planteamiento de directrices, método y condiciones tendientes 
a la transformación ordenada y paulatina del sistema educativo en su conjunto". De tal forma, que esta logre impactar en sus investigaciones, los paradigmas de aprendizaje articulados a los cambios generados desde una "revolución científica y una educación holística".

Otro trabajo que se logra ubicar dentro de la revisión de la literatura es el de Sancho, Ornellas \& Arrazola (2018), que presenta la situación cambiante de la universidad en la era digital, donde señala cómo el avance de las tecnologías ha abierto nuevos desafíos a la universidad en términos de la construcción de conocimiento y en la consolidación de sociedad. Actualmente, la universidad se ha trasladado a escenarios extramurales como es la virtualidad, por lo que tanto docentes como estudiantes deben asumir nuevas formas de enseñar y aprender en sintonía con los nuevos repertorios tecnológicos. Sin embargo, la mirada hacia estos nuevos episodios educativos debe ser critica para ubicar el sentido de la universidad actual.

Sin duda la revisión teórica que se ha realizado permite referir que la universidad debe reinventarse y cuestionar su misión en función de los intereses y necesidades del contexto y los sujetos que en ella confluyen. De otra parte, es importante entender que la esencia de la universidad debe estar soportada en acciones científico humanistas que cuestionen cómo se está asumiendo la formación de profesionales en términos contemporáneos para develar el sentido de la universidad como un agente de transformación y su contribución.

\section{METODOLOGÍA}

El desarrollo de este análisis se sustenta en la metodología de investigación de teoría fundamentada que según Strauss (1967), es un método de investigación donde la teoría emerge de los datos. Esta metodología es pertinente ya que permite develar aspectos relevantes de una determinada área de estudio. Según, Glaser (1992), la teoría fundamentada es útil para investigaciones en campos que conciernen a temas relacionados con la conducta humana dentro de diferentes organizaciones, grupos de otras configuraciones sociales, brindando la posibilidad de profundizar sobre el fenómeno que se desea indagar. Lo más valioso de esta metodología es su pertinencia al momento de construir teorías, conceptos y proposiciones partiendo directamente de los datos y no de supuestos a priori. La revisión de literatura se realizó haciendo una clasificación teórica, seguido de un análisis de documentos para luego proceder a la escritura del texto.

\section{DESARROLLO}

Es claro que en Colombia hablar de universidades emergentes en el siglo XXI, es visibilizar resultados de pruebas y rankings internacionales de investigación, de ahí que se cataloga que la que cumple estos criterios es la Universidad de los Andes según las investigaciones Thompson Reuter en el 2018, la cual ocupa el puesto 17 de 100 , mientras que otras universidades del país no alcanza a superar estos niveles. Lo cierto es que en el mundo la Universidad de Bejing ocupa el primer lugar en universidades emergentes y la universidad de Sao Paulo en Brasil por encima de otras en Latinoamérica, que eran tradición en estos resultados, como lo muestra la Revista Dinero de septiembre de 2018. Pero hay otras circunstancias, que deben propiciarse al valorar estos resultados y no solo valorar los rankings, sino los procesos humanizadores en ellas, las transformaciones y el cambio social que dinamizan la cultura, la ciencia y el propio pensamiento que incide en el cambio de paradigmas emergentes a nivel universitario y en la formación de sujetos sociales con visión de realidad y dar un mayor sentido global del ser, aquello que se viene desarrollando y reflexionando al asumir un nuevo humanismo e 
ir más allá de las problemáticas económicas apu ntando a un paradigma más crítico.

Esto significa que la universidad debe ser un escenario que permita la interacción de las disciplinas, las prácticas conjuntas y la reflexión compartida de una sociedad culturalmente diversa, donde se supere la teoría para abrir paso a la práctica. Hay un malestar universitario derivado de una universidad donde prima la condición económica, si bien los aspectos políticos y sociales juegan un papel preponderante en la construcción de universidad, también los movimientos sociales quienes vislumbran una universidad popular distanciada de patrones culturales que se encasillan en prioridades capitalistas, y que conducen a la crisis de la universidad en el siglo XXI.

La universidad pareciera que dejó de ser un escenario consensuado que se construye con la participación popular, a pasar a un sistema de educación superior donde cada día prima más los intereses económicos que académicos, y las necesidades de la sociedad pasan a un segundo plano, se puede ver en la actualidad que por un lado van las reformas y por otro las necesidades de las comunidades, al parecer todo depende del ministro de turno.

Sin duda los desafíos de la universidad son muchos, ¿cómo atender la demanda social, con un limitado apoyo económico? ¿cómo defender la investigación, cuando la ciencia está sujeta a la inversión del estado?, ¿acaso la universidad pública perdió la lucha?, ¿cambió sus prioridades?, ¿se volvió una mercancía académica? ¿perdió autonomía?, ¿perdió autoridad? ¿acaso la universidad se descompuso?, ¿no habrá forma de componerla? Son muchos los cuestionamientos que emergen al momento de analizar la situación actual de las universidades. Es preocupante ver cómo la crisis financiera ha afectado la autonomía de la universidad pública, por esta razón es necesario pensar en una universidad distanciada del mercado universitario y soportada en el sentir real de la academia y su compromiso social.

Otra problemática aunada a las planteadas anteriormente, es encontrarse con un sistema universitario segmentado, donde pierde valor el título universitario dependiendo la disciplina que certifica y donde el docente es situado como un todopoderoso que debe responder por procesos de docencia, extensión, investigación, internacionalización, eventos, tutorías, evaluaciones, entre otras actividades académicas que hacen parte del diario vivir de la universidad y que no dan espera.

El llamado es a pensar sobre ¿cómo responder a los problemas que enfrenta la universidad de hoy?, y más aún cuando en el marco de la globalización las TIC cobran un papel importante postulando la llamada hoy sociedad de la información y del conocimiento, situando nuevas fuentes de información y abriendo novedosos escenarios de participación que permiten la construcción colectiva, sin duda todos estos cambios han impactado en el quehacer propio de la universidad como en la enseñanza, la investigación y la extensión; pero vale la pena cuestionar también el impacto que las TIC han generado en aquellos escenarios donde su llegada a demorado abriendo brechas digitales que apartan la participación de estas comunidades.

Sin duda se escasea de reformas que defiendan la universidad pública y que estén sintonizadas con las necesidades de los sujetos que allí confluyen, al parecer las reformas educativas avanzan por un lado y las realidades educativas por otro; provocando un distanciamiento, entre lo que se establece como norma y lo que realmente se necesita. En este sentido el estado y la universidad en el marco de un contrato social deben proponer reformas que respondan a las demandas sociales, pero entendiendo que no hay soluciones nacionales 
o genéricas, sino que los problemas se deben intervenir teniendo presente el contexto, por lo que las políticas deben regionalizarse para atender las necesidades sentidas de una comunidad local. Por otra parte, se puede ver hoy el débil apoyo a la investigación aun cuando es la puerta de desarrollo de la sociedad; es preocupante ver que en la universidad de hoy se concursa por un apoyo económico para sacar adelante un proyecto de investigación; es decir, las problemáticas y las necesidades sociales se volvieron un concurso que dependiendo el problema a investigar es el apoyo económico que se le asigna. Morin (1994), argumenta que la ciencia se ha vuelto ciega por su incapacidad de concebir su rol social y su negligencia para articular las relaciones multidisciplinarias del contexto. La complejidad debe entenderse como la oportunidad para revelar un conocimiento más rico y menos ciego.

Sin embargo, la tarea de la universidad no es solo identificar los desafíos que emergen de la sociedad, sino ver su capacidad de enfrentarlos para ofrecer procesos de intervención in situ que permitan mejorar el tejido social. En este sentido, cobra importancia, la movilización de prácticas, saberes y discursos que se congregan en la universidad con el fin de responder a las demandas de una modernidad cada vez más compleja donde en ocasiones prevalece la productividad empresarial antes que la academia con sentido social. Mejía (2010), reconoce que el desarrollo de la ciencia, es un hecho prestigioso que penetra la enseñanza, la industria y la vida; sin embargo, en ocasiones la ciencia se margina y se reduce a la mera aplicación práctica del conocimiento, dejando de lado la formación humana del sujeto, que sin duda espera recibir una formación integral que le permita proyectar un futuro enmarcado en la justicia social.

Es fundamental reflexionar como la universidad del siglo XXI, en su mayoría, en América
Latina, genera una serie de reformas internas que a la larga no solucionan sus problemas de fondo, pues pone a la academia al servicio de la administración y no la administración al servicio de la academia. Esta situación sin duda afecta el sentido misional de la universidad en la academia, investigación y extensión.

Las universidades que más han crecido son las universidades privadas, mientras las universidades públicas han estado en manos de los políticos de turno que no la dejan crecer y se colocan al servicio de la politiquería, falta seriedad en los procesos de construcción de las universidades públicas, son ya 26 años de la promulgación de la ley 30 de 1992, en la que los gobiernos deberían tener una responsabilidad social, y no la han asumido verdaderamente como debe ser, el mismo sistema que las creó las ha vuelto insostenibles, es una universidad en crisis económica, por cuanto asumió cobertura pero no proyectó sus consecuencias, claro orquestado por los gobiernos de turno que cada vez se lavan las manos y dejan a la universidad privada en crecimiento y la universidad pública en decadencia, esta responsabilidad social implica que hoy la universidad está desfinanciada y desconoce su propia realidad para lo que fueron constituidas, en pro de la construcción de un eje articulador desde la base académica y no economicista.

La universidad del siglo XXI necesita de políticas educativas eficientes, que sean de Estado y no de gobierno, ya que sus emergencias requieren ser el motor que movilice la ciencia, la tecnología, la cultura de manera transparente y coherente a los cambios emergentes de la sociedad, esta debe permitirse pensar, crecer y formar hombres y mujeres capaces de transformar la academia, la investigación, proyectarse, tomar decisiones y atreverse a generar cambios fundamentales que permitan repensar la universidad que requiere América Latina y el tipo de formación de intelectuales que está llamada a ofrecer. Tapia 
(2007), señala que "Reconstruir la comunidad en la universidad significa transformar un gobierno de carácter nepotista y discrecional en un gobierno que tenga, por un lado, participación social y participación de la comunidad en tanto tal". (p.46).

En este sentido, se hace necesario dejar la ingobernabilidad en las políticas públicas educativas de los gobiernos de cada país y hacer de la gobernabilidad un sustento de las políticas públicas eficientes y de calidad, al servicio de la academia y sus avances, brindar los escenarios de participación y democrática universitaria desde las bases y como prioridad sustentada en una sociedad que exige cambios estructurales ya sea en la ciencia, la tecnología, la innovación, la búsqueda de mejores talentos y como lo señala Fernández (2014) se requiere en la actualidad una universidad que responda a los requerimientos políticos, sociales y académicos, tanto de largo plazo, como más inmediatos. Es de esperar que estos debates posibiliten estructurar entre todos, un nuevo proyecto de Reforma Universitaria que -como homenaje a los estudiantes de Córdoba de 1918refleje los lineamientos para atender dichos requerimientos. Ojalá esta nueva propuesta de Reforma Universitaria para el siglo XXI pueda elaborarse antes de alcanzar -ya muy pronto- el centenario de la Reforma Universitaria.

Así mismo, se necesita que la universidad asuma su responsabilidad social y política, y responda a los retos que la sociedad exige, desde la promoción de prácticas democráticas libres y participativas que permitan contribuir desde la academia e investigación en dotar a los estudiantes de herramientas y estrategias para mejorar sus condiciones de vida, trabajar en pro del posconflicto con coherencia $y$ calidad educativa. Se trata entonces de dar una responsabilidad social a la universidad, comprender que, bajo su legitimidad, logra una verdadera producción de conocimiento y un sentido más humano como alternativa a una verdadera institucionalidad y a sus desafíos, como formas de mejorar la democratización, su emancipación y flexibilidad, en sus procesos acorde a las funciones para las cuales ha sido reconstruida, como sustento de la construcción de nación.

\section{AGRADECIMIENTOS}

Los autores expresan su agradecimiento a la Universidad Pedagógica y Tecnológica de Colombia y a la Licenciatura en Tecnología por brindar los escenarios de indagación y contribuir con el proceso de investigación adelantado que seguramente contribuirá con los procesos de reflexión que se generan en torno a la llamada crisis de la universidad.

\section{CONCLUSIÓN}

Vale la pena repensar la universidad en tiempos difíciles desde una mirada política, antropológica y pedagógica, ya que la formación de profesionales va más allá del aspecto meramente disciplinar, sino que se debe pensar en un profesional con sentido social. La consigna central de la universidad debiera ser el pensar en una universidad para el cambio, que se conciba como un ecosistema social donde las fronteras del conocimiento desaparecen posibilitando la acción social y el trabajo conjunto, cuya misión está orientada a contribuir con una sociedad más justa, vinculada a la ética y con conciencia ciudadana, que permita la reflexión críticoconstructiva en las aulas, atacando por todos los medios problemáticas como las prácticas de corrupción desde la administración y academia, la capacitación de profesores en manejos de problemas sociales como lo son: la violencia intrafamiliar, la drogadicción, el suicidio, el narcotráfico y la falta de equidad que conlleva a profundizar la pobreza, entre otros; de esta manera poder incidir en la calidad educativa para transformarse hacia una verdadera calidad de vida de sus participantes. 


\section{REFERENCIAS BIBLIOGRÁFICAS}

- Bernheim, C. T. (2003). La universidad ante los retos del siglo XXI. UADY.

- Bruner, J. (1990). La educación superior en América Latina, Santiago de Chile, Fondo de Cultura Económica.

- Brunner, J. J. (Coord.) (1995). Educación superior en América Latina: una agenda de problemas, políticas y debates en el umbral del año 2000. Bogotá: Universidad Nacional de Colombia.

- Castells, M. (2003). Panorama de la era de la información en América Latina: ¿Es sostenible la globalización? En: F. Calderón (coord.), ¿Es sostenible la globalización en América Latina? Santiago de Chile: Fondo de Cultura Económica.

- Clark, B., \& Neave, G. (1992). Encyclopedia of Heigher Education (Vol. IV).

- Corrales, S. (2007). La misión de la universidad del siglo XXI. Razón y palabra. no. 57 (junio-julio). México: Universidad de la Comunicación México D.F.

- Dewey, J. (1989). Cómo pensamos. Nueva exposición de la relación entre pensamiento reflexivo y proceso educativo Paidós, Barcelona, $249 \mathrm{pp}$

- De la Red V. N. (2009). Necesidades emergentes y responsabilidad social universitaria. Departamento de Sociología y Trabajo Social, en Revista Alternativas de trabajo social $N^{\circ} 16$ [65-76], Universidad de Alicante, Facultad de Educación y Trabajo social. Universidad de Valladolid.

- De Sousa S., B. (2004) La universidad en el siglo XXI. Para una reforma democrática y emancipadora de la universidad. Madrid y Buenos Aires: Miño y Dávila, Laboratorio de Políticas Públicas.
- De Sousa, S. B. (2007). La Universidad en el siglo XXI. Para una reforma democrática y emancipatoria de la universidad. CIDESUMSA, ASDI y Plural editores, La Paz, Bolivia.

- Fernández L., N. (2014). Universidad, sociedad y conocimiento. Reflexiones para el debate. Avaliação, Campinas; Sorocaba, SP, v. 19 , n. 3, p. 663-687, Universidad Nacional de Tres de Febrero. Buenos Aires, Argentina.

- Kerr, C. (1963). The Uses of the University, Harvard University Press, Cambridge.

- Glaser, B. \& Strauss, A. (1967). The discovery of grounded theory. Chicago: Aldine Press

- Glaser, B.G. (1992). Basic of grounded theory analysis. Emerge us. Foreign.Mill Valley. CA. Sociology Press. California.

- Martínez P, J., \& Abadía G. C., \& Montenegro, L. (2016). Urdimbre sobre la universidad en la mercantilización del saber: Paul Ricoeur, Jacques Derrida, Boaventura de Sousa Santos. Tabula Rasa, (24), 381-401.

- Mejía, J. (2010). Los Movimientos de proyectos educativos universitarios en un contexto histórico de la vida colombiana, en la construcción del Estado-Nación a finales del siglo XIX y comienzos del siglo XX. Revista Polémica, 134-155.

- Morin, E., \& Pakman, M. (1994). Introducción al pensamiento complejo. Barcelona, Gedisa.

- Ortiz, A. (2017). La universidad emergente del siglo XXI. Pedagogía currículo y didácticas en la educación superior. Madrid, Editorial Academia Española. 
- Pujadas (s.f). Los desafíos que hoy enfrenta la universidad. Recuperado de: http://200.16.86.50/Digital/658/revistas/vsi/ pujadas10-10.pdf

- Samper, S. Z. (2007). Universidad, crisis y nación en América Latina. Revista De Estudios Sociales, (26), 148-157.

- Sancho., G. J, Ornellas, A. \& Arrazola, J. (2018). La situación cambiante de la universidad en la era digital. Universidad de Barcelona España.

- Tapia, L. (2007). Debate sobre la universidad del siglo XXI. Recuperado de: http://biblioteca.clacso.edu.ar/clacso/ coediciones/20160304040311/cap2.pdf 